\title{
A SURVEY OF THE AMPHIBIAN FAUNA OF KERALA - DISTRIBUTION AND STATUS
}

\author{
M.I. Andrews ${ }^{1,2}$, Sanil George ${ }^{3}$ and Jaimon Joseph ${ }^{1}$ \\ 1 Department of Zoology, Mar Thoma College, Tiruvalla, Kerala 689103, India \\ ${ }^{2}$ RGCB, Trivandrum, Kerala 695014, India \\ E-mail: ${ }^{1}$ mipeandrews@yahoo.co.in, ${ }^{3}$ rhacophorus@rediffmail.com
}

web supplement

\begin{abstract}
A total of 70 species of amphibians belonging to 20 genera of seven families and two orders were recorded during a survey for amphibian fauna of Kerala from August 1999 to August 2002. Forty-nine of these species recorded are endemic to Kerala or the Western Ghats. Most districts in the state have rich amphibian fauna with Wayanad district having the highest number of species and Kozhikkode district the lowest number of species. Altitudinal distribution showed that the highest number of species occurred between 500$1000 \mathrm{~m}$ and the lowest number in areas above $1500 \mathrm{~m}$. Information on the habitats and status of amphibians in the state are also provided.
\end{abstract}

KEYWORDS

Amphibian fauna, distribution, status, endemism, Kerala

The Indian subcontinent, particularly the Western Ghats and the Eastern Himalaya harbour a rich amphibian fauna. The diversified topography, geographic location, high rainfall and humidity have likely supported the survival of numerous species of amphibians here. Amphibian fauna of India comprises of about 225 species (Biju, 2001), however the precise number of species is not known since new frogs are being discovered and with many being elevated to species status from synonymy at a remarkable rate. The south Indian State of Kerala is known to hold a high number of amphibian species; 87 species have been reported from here (Andrews \& George, 1998). The recent discovery of a Pig-nosed Frog Nasikabatrachus sahyadrensis (Biju \& Bossuyt, 2003) from the region, which has close affinities with the Family Sooglossidae found in the Seychelles Archipelago in the Indian Ocean, highlights the amphibian faunal wealth in the state.

Studies on the amphibian fauna of Kerala date back to fauna volumes published by Boulenger (1890, 1892), and Ferguson (1904) who listed batrachians of Travancore. Most other studies on amphibians in the state are however limited to short surveys and new descriptions (Pillai, 1978, 1981, 1986; Pillai \& Pattabiraman, 1981, 1990; Inger et al., 1984a,b; Ravichandran \& Pillai, 1990; Andrews, 1994; George, 1995; George et al., 1996; Zacharias \& Bhardwaj, 1996; Radhakrishnan, 1996a,b; Radhakrishnan et al., 1996; Easa, 1998; Abraham \& Easa, 1999; Radhakrishnan \& Ravichandran, 1999; Oommen et al., 2000; Abraham et al., 2001; Dubois et al., 2001; Biju \& Bossuyt, 2003). Hence, the present work was undertaken to make an extensive study of the amphibian fauna of Kerala with special reference to their distribution and status.

\section{Study ARea AND Methods}

Kerala $\left(8^{0} 17^{\prime} 30^{\prime \prime}-12^{0} 47^{\prime} 40^{\prime \prime} \mathrm{N} \& 74^{0} 51^{\prime} 57^{\prime \prime}-77^{0} 24^{\prime} 47^{\prime \prime} \mathrm{E}\right)$ is one of the smallest states of India; with an area of $38,590 \mathrm{~km}^{2}$ it is only
$1.18 \%$ of the total area of India. Flanked by the Western Ghats on the eastern and the Arabian Sea on the western side, Kerala receives high rainfall with an average of $2,963 \mathrm{~mm}$ (Iype et al., 1991), and mainly from the south-west monsoon (JuneSeptember; 60\%), the rest from the north-east monsoon (October-December). The mean annual temperature varies from $25.4^{\circ} \mathrm{C}$ to $31^{\circ} \mathrm{C}$.

The present study was carried out from August 1999 to August 2002, during which visits to several parts of Kerala and to all the protected areas in the state were made. Field studies and samplings were mainly done during the monsoon period while on few occasions sampling was also done during the pre-and post-monsoon period. The altitude in the state was categorised as $0-500 \mathrm{~m}, 500-1000 \mathrm{~m}, 1000-1500 \mathrm{~m}$ and above $1500 \mathrm{~m}$ for sampling across altitudes. At all localities visited, intensive search for amphibians for direct visual encounter were made, for six hours daily between 0600 to $0900 \mathrm{hr}$ and 1700 to $2000 \mathrm{hr}$. Here all potential sites such as near water bodies, along streams, under leaf litter, on tree trunks and on foliage were searched. On every amphibian encounter, information on species, habitat and altitude were recorded. All amphibians observed during the study were photographed (see images in the web supplement ${ }^{\mathrm{w}}$ ). Where possible amphibians were collected and only one male and a female for most species were preserved in $6 \%$ formalin for further taxonomic studies in the laboratory. These are deposited in the Zoology Museum of Mar Thoma College in Tiruvalla, Kerala. In most cases specimens were released after taking morphometric measurements in the area from where collected. Species identification was made based on literature and with help from other taxonomists.

\section{Findings of the Survey}

Of the 87 amphibian species reported from the State (Andrews \& George, 1998), a total of 70 species of amphibians belonging to 20 genera of seven families and two orders were recorded from various parts of Kerala during this survey (Tables $1 \& 2$ ). Forty-nine of the 70 species recorded during the study are endemic to Kerala or the Western Ghats. The highest number of amphibian species was recorded from Wayanad district (41 species), while the lowest number of species was observed in Kozhikkode district with only 11 species.

Two records of amphibians not reported from Kerala previously were found during this study. Minervarya syhadrensis was originally described based on specimens collected in Maharashtra, and as subspecies of Rana limnocharis, which was raised to species rank by Dubois (1984). Biju (2001) reported this species from the Western Ghats but was not recorded from

www.zoosprint.org 
Kerala. Several specimens of this species were recorded from Malapuram and Kasaragod districts during the study. Micrixalus phyllophilus first discovered from Tamil Nadu was found during the study. A single specimen was recorded from Kurichermala, Wayanad.

Ansonia rubigina, Nyctibatrachus aliciae and Micrixalus gadgili that have only been recorded on few occasions by earlier workers in the state were also recorded during the study, and again only from a few places. Micrixalus nudis, Nasikabatrachus sahyadrensis, Nyctibatrachus minor, Polypedates pseudocruciger and Rhacophorus lateralis, earlier recorded only from a few places were either found in many places in the state or many specimens were observed during the study. Some species -- Bufo hololius, B. silentvalleyensis, Indirana leithii, Melanobatrachus indicus, Micrixalus thampii, Nannobatrachus beddomii, Pedostibes tuberculosus, Philautus travancoricus and Rhacophorus calcadensis known only from their type localities or based on a few specimens collected by one or two workers in the state earlier could not be located during this survey.

\section{Altitudinal Distribution}

The highest number of species was found between 500 and $1000 \mathrm{~m}$ altitude (48) and the lowest number of species above $1500 \mathrm{~m}$ (7), while 39 species were recorded between 0 and $500 \mathrm{~m}$ and 35 species between 1000 and $1500 \mathrm{~m}$ with some species overlapping in their altitude range. The seven species found to occur above $1500 \mathrm{~m}$ were also found to occur in other areas, and no amphibian species were found exclusively in the region above $1500 \mathrm{~m}$. Species like Indirana beddomii, Nyctibatrachus major and Rana temporalis were recorded from all the altitudes indicating their diverse adaptations to live in the low and high altitudes. Details of the altitude occupancy of each species is given in Table 1.

\section{Habitat Occupancy}

The highest number of amphibian species (41) was found in agricultural fields and plantations while least number of species (9) was found in grasslands. The evergreen and semi-evergreen forests harbored 39 species, the deciduous forests supported 28 and the sholas had 18 species. Some species were observed to inhabit more than one habitat type, while 29 species were observed in only one habitat (Table 1).

Bufo melanostictus was found to occur in all five habitats while species like Indirana beddomii and Rana curtipes were recorded in all habitats except agricultural fields and plantations. Euphlyctis cyanophlyctis, Fejervarya keralensis and $F$. limnocharis were seen in all habitats except sholas; Rana temporalis was present in all habitats except grasslands. Several species were found exclusively in agricultural fields and plantations while Gegeneophis carnosus, Micrixalus gadgili, M. phyllophilus, Nyctibatrachus aliciae, N. beddomii, Philautus nasutus, P. temporalis and Uraeotyphlus oxyurus were seen only in the evergreen and semi-evergreen forests; Bufo scaber and Philautus glandulosus were present only in the deciduous forests and Philautus tinniens only in the sholas.
Forty-five of the 70 amphibians recorded were non-riparian species, 15 were riparian and 10 were intermediate species. All caecilians, bufonids, microhylids and rhacophorids were nonriparian species. Among the ranids Indirana brachytarsus, I. diplostictus, I. semipalmatus, Rana malabarica, Sphaeroteca breviceps, S. rolandae and S. rufescens were non-riparian species while several others were riparian species and few were intermediate species. Among bufonids, only Ansonia rubigina was found to be a riparian species.

\section{Status of Amphibians}

Eighteen species were seen commonly, while all other species (52) were found rarely in the state. Among the rare species, Uraeotyphlus interruptus, U. oxyurus, Uperodon systoma Micrixalus gadgili, M. phyllophilus, Nyctibatrachus beddomii, Philautus tinniens, P. glandulosus, Rhacophorus lateralis and Sphaeroteca rolandae were recorded from only one locality each. The commoner species were observed to occur in less number in some places while they were numerous in other parts of the state. The most abundant family observed during the survey was Ranidae with representatives from 8 genera (31 species), followed by the families Rhacophoridae and Microhylidae with members from 3 genera (16) and 4 genera (8) respectively. Philautus was the most abundant genus (12), followed by Micrixalus (6), Fejervarya (5) and Indirana (5).

It is most likely that we may have missed some species during the field surveys, as we could not cover the entire state within the study period. Further surveys and studies need to be taken up in many parts of Kerala to get a better and complete picture of the amphibian fauna of the state.

\section{REFERENCES}

Abraham, S.K. and P.S. Easa (1999). Addition to the amphibians of Aralam Wildlife Sanctuary, Kerala. Cobra 38:12-13.

Abraham, S.K., P.S. Easa, S.A.S. Jahas and C.P. Shaji (2001). Amphibian fauna of Wayanad, Kerala. Zoos' Print Journal 16(4): 457461.

Andrews, M.I. (1994). Studies on amphibians of Kuttanad, a natural wetland in Central Kerala. Final Report of the Project sponsored by STEC, Kerala.

Andrews, M.I. and S. George(1998). Amphibian resources of Kerala. Cobra 33: 41-48.

Biju, S.D. (2000). Two frogs and hundred years. Biodiversity India, ISCB Newsletter 8-12:10-11.

Biju, S.D. (2001). A synopsis to the frog fauna of the Western Ghats, India. Occasional Publication of the Indian Society for Conservation Biology 1: 24pp.

Biju, S.D. and F. Bossuyt (2003). New frog family from India reveals an anicient biogeographical link with the Seychelles. Nature 425: 7117 .

Boulenger, G.A. (1890). The fauna of British India, including Ceylon and Burma- Reptilia and Batrachia, Tayler and Francis, Xviii+pp 541. Boulenger, G.A. (1891). Description of a new species of frog obtained by Mr. H.S. Ferguson in Travancore, South India. Journal of the Bombay Natural History Society 6: 450.

Boulenger, G.A. (1892). Description of a new toad from Travancore (Bufo scaber). Journal of the Bombay Natural History Society 7: 317318.

Boulenger, G.A. (1883). Description of new species of reptiles and batrachians in the British Museum. Annual Magazine of Natural History, 5(12): 161-167. 


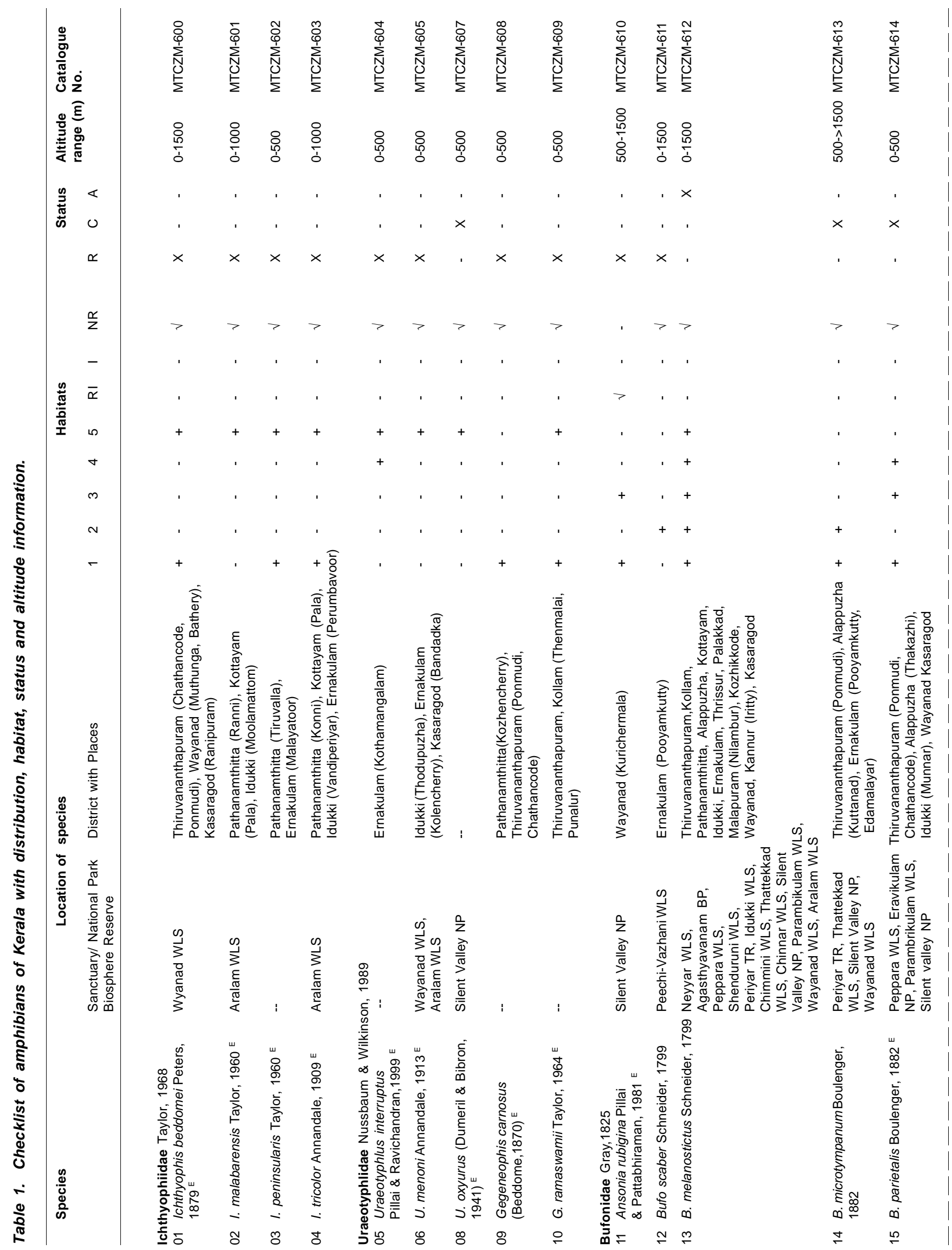




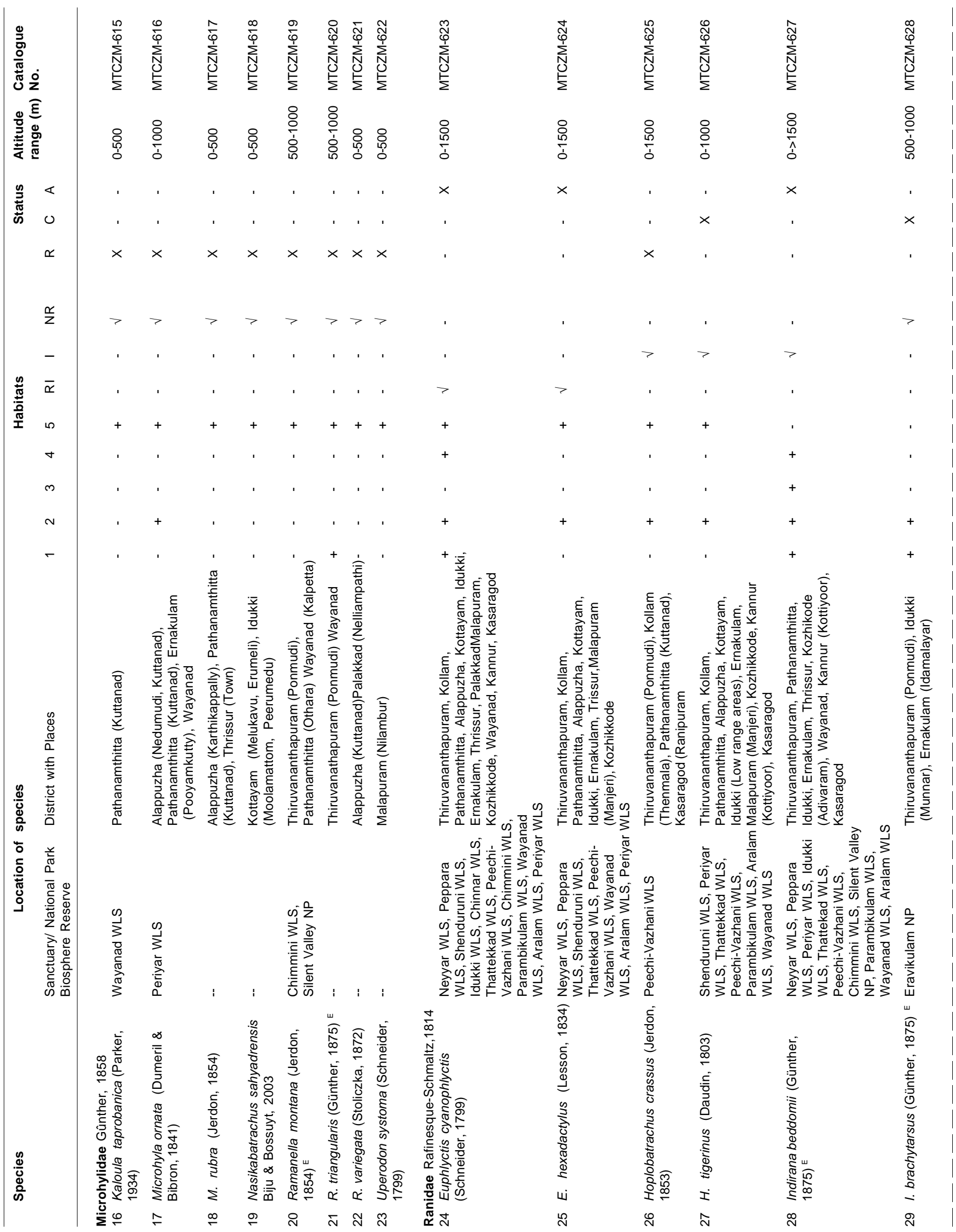




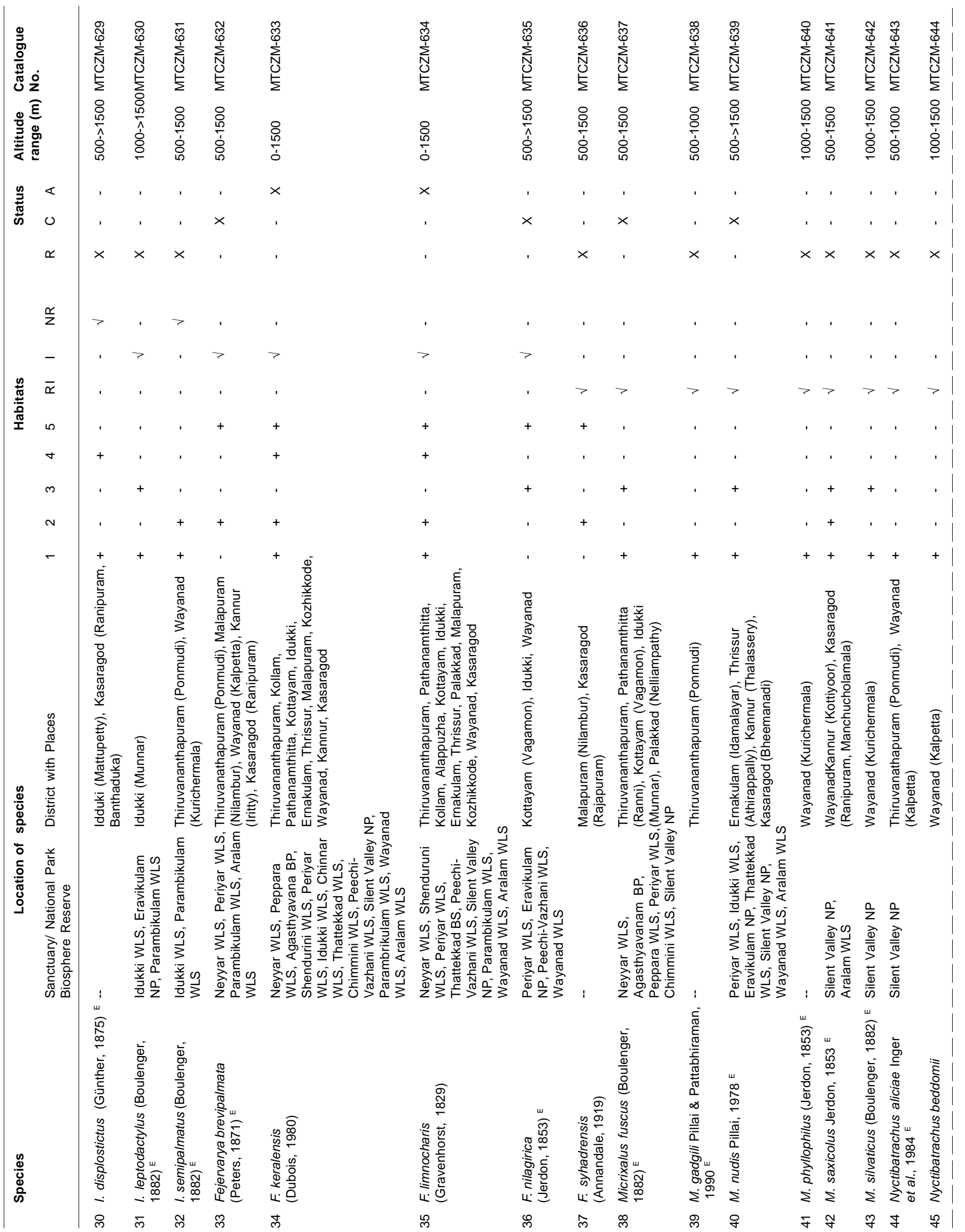




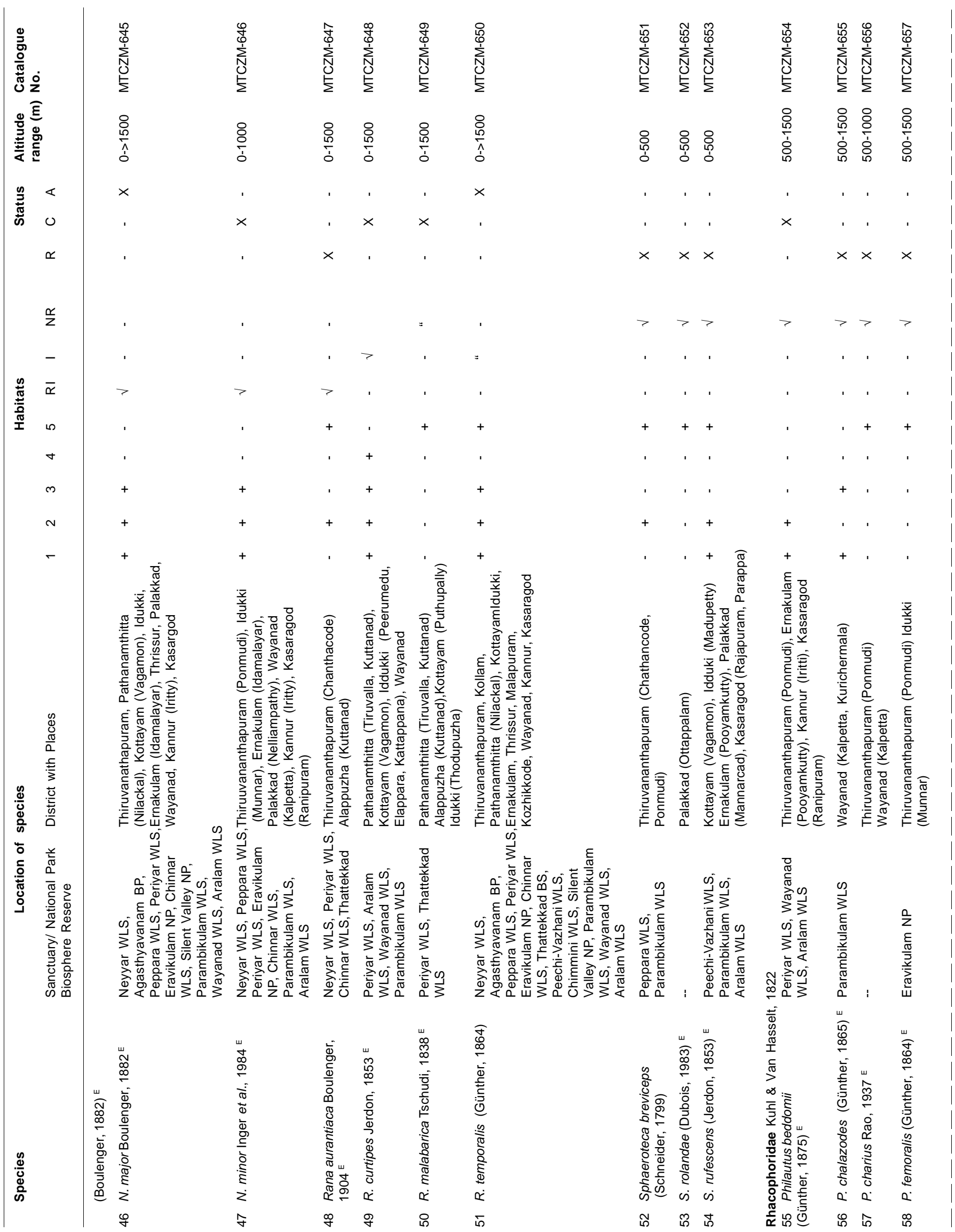




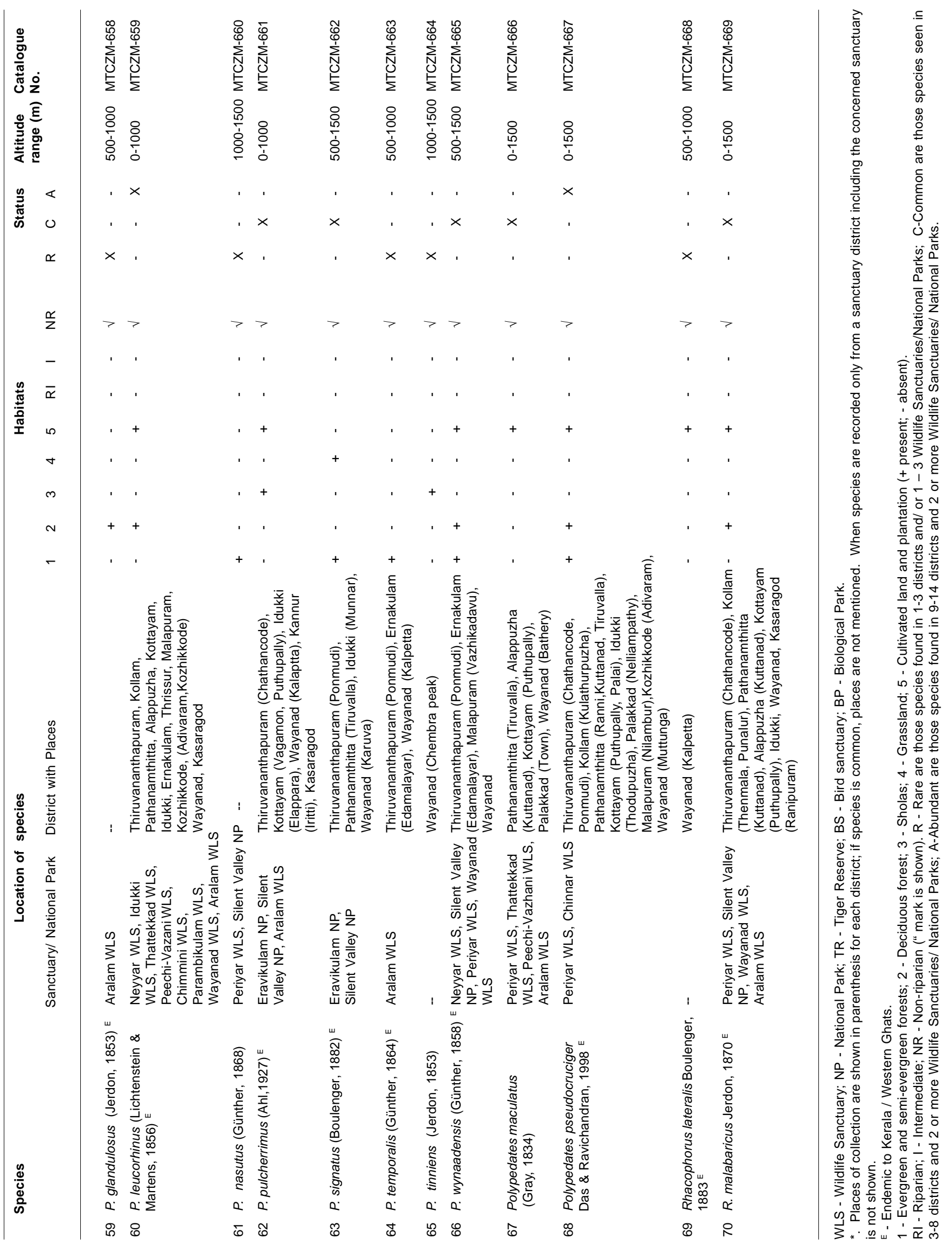


Table 2. District-wise distribution of amphibians of Kerala

\begin{tabular}{|c|c|c|c|c|}
\hline \multicolumn{2}{|c|}{ SI. No. Species } & Habitat & Microhabitat & Status \\
\hline \multicolumn{5}{|c|}{ a) Thiruvananthapuram district $\left(8^{0} 17^{\prime}-8^{0} 54^{\prime} \mathrm{N} \& 7^{\circ} 4^{\prime}-77^{0} 17^{\prime} \mathrm{E}\right)$} \\
\hline 1 & Ichthyophis beddomei & $\mathrm{FS}, \mathrm{CL}$ & under wet humid black soil & rare \\
\hline 2 & Gegeneophis carnosus & $\mathrm{FS}, \mathrm{CL}$ & under wet humid black soil & rare \\
\hline 3 & G. ramaswamii & MDF, CL & under soil & common \\
\hline 4 & Bufo melanostictus & EGF, SEF, MDF, CL & on bare soil, under stones and crevices & common \\
\hline 5 & B. microtympanum & $\mathrm{MDF}, \mathrm{CL}$ & under stones & rare \\
\hline 6 & B. parietalis & EGF, SEF & forest floor-on leaf litter & rare \\
\hline 7 & Ramanella montana & EGF & on wet soil near water pools & rare \\
\hline 8 & R. triangularis & EGF & in tree holes & rare \\
\hline 9 & Euphlyctis cyanophlyctis & MDF, CL & floats on water surfaces & very common \\
\hline 10 & E. hexadactylus & MDF, CL & in water-hides among submerged plants & common \\
\hline 11 & Hoplobatrachus crassus & TP & on wet soil/near water & rare \\
\hline 12 & H. tigerinus & $\mathrm{CL}$ & in marshes & common \\
\hline 13 & Indirana beddomii & MDF, SEF, EGF & forest floor - on wet soil or leaf litter and under stones & common \\
\hline 14 & I. brachytarsus & EGF & forest floor-on leaf litter & rare \\
\hline 15 & I. semipalmatus & FS & on grass -covered rocks & rare \\
\hline 16 & Fejervarya brevipalmata & MDF & among grasses in marsh & rare \\
\hline 17 & F. keralensis & SEF, MDF, TP & stream banks- on wet sand / soil and leaf litter & common \\
\hline 18 & F. limnocharis & SEF, MDF, TP & in marshes and wet lands among grasses & common \\
\hline 19 & Micrixalus fuscus & EGF, SEF & stream banks and stream beds -on boulders or wet soil & common \\
\hline 20 & M. gadgili & EGF, SEF & stream banks and beds & rare \\
\hline 21 & Nyctibatrachus aliciae & EGF & hill streams-in water & rare \\
\hline 22 & N. major & EGF, MDF & streams-submerged in water & common \\
\hline 23 & N. minor & EGF, DDF & in very shallow streams; on wet soil, under stones and on leaf litter & common \\
\hline 24 & Rana aurantiaca & DDF & in marsh - among grasses, in water or on wet soil & rare \\
\hline 25 & R. temporalis & EGF, SEF, MDF, CL & close to water among grasses in marshes & very common \\
\hline 26 & Sphaerotheca breviceps & CL inside MDF & marshy soil & rare \\
\hline 27 & Philautus beddomii & $\mathrm{GL}$ & on herbs & rare \\
\hline 28 & P. charius & EGF & on herbs & rare \\
\hline 29 & P. femoralis & $\mathrm{GL}$ & on herbs & rare \\
\hline 30 & P. leucorhinus & $\mathrm{FS}, \mathrm{CL}$ & on herbs and shrubs & common \\
\hline 31 & P. pulcherrimus & DDF & on the leaf of shrubs & rare \\
\hline 32 & $P$. signatus & $\mathrm{GL}$ & on grass leaf & rare \\
\hline 33 & P. temporalis & $\mathrm{GL}$ & on herbs & rare \\
\hline 34 & P. wynaadensis & $\mathrm{GL}$ & on herbs & rare \\
\hline 35 & Polypedates pseudocruciger & DDF & on shrubs in marshes & rare \\
\hline 36 & Rhacophorus malabaricus & $\mathrm{CL}$ & on the stem of shrubs & rare \\
\hline 37 & Bufo beddomii & - & - & - \\
\hline 38 & Indirana diplostictus ${ }^{*}$ & - & - & - \\
\hline 39 & Micrixalus nudis * & - & - & - \\
\hline 40 & Nannobatrachus beddomii & - & - & - \\
\hline 41 & Pedostibes tuberculosus* & - & - & - \\
\hline \multicolumn{5}{|c|}{ b) Kollam district $\left(9^{0} 28^{\prime}-8^{\circ} 45^{\prime} \mathrm{N} \& 76^{\circ} 28^{\prime}-77^{0} 17^{\prime} \mathrm{E}\right)$} \\
\hline 1 & Gegeneophis ramaswamii & $C L$ & under soil & rare \\
\hline 2 & Bufo melanostictus & MDF & forest floor-on bare soil & common \\
\hline 3 & Euphlyctis cyanophlyctis & MDF, CL & in water & very common \\
\hline 4 & E. hexadactylus & $\mathrm{CL}$ & in water & very common \\
\hline 5 & Hoplobatrachus crassus & MDF & in small water pools & rare \\
\hline 6 & H. tigerinus & $\mathrm{CL}$ & among submerged plants in water & common \\
\hline 7 & Fejervarya keralensis & SEF, MDF, TP & close to water - on wet soil, leaf litter and under stones & common \\
\hline 8 & F. limnocharis & MDF, CL & among wet grasses and on leaf litter & common \\
\hline 9 & Rana temporalis & MDF & streams- in water, on wet soil and boulders & common \\
\hline 10 & Philautus leucorhinus & MDF & on shrubs & common \\
\hline 11 & Polypedates pseudocruciger & $\mathrm{CL}$ & on shrubs & rare \\
\hline 12 & Rhacophorus malabaricus & $C L$ & on shrubs & rare \\
\hline \multicolumn{5}{|c|}{ c) Pathanamthitta district $\left(9^{0} 37^{\prime}-9^{0} 53^{\prime} N \& 7^{0} 55^{\prime}-76^{0} 83^{\prime} E\right)$} \\
\hline 1 & Ichthyophis malabarensis & $\mathrm{CL}$ & under soil & rare \\
\hline 2 & I. peninsularis & $\mathrm{CL}$ & under soil & rare \\
\hline 3 & I. tricolor & SEF, CL & under soil & rare \\
\hline 4 & Gegeneophis carnosus & $\mathrm{CL}$ & under soil & rare \\
\hline 5 & Bufo melanostictus & $\mathrm{CL}, \mathrm{SEF}$ & on bare soil or litter & common \\
\hline 6 & Kaloula taprobanica & SEF, CL & under decayed leaves & rare \\
\hline 7 & Microhyla ornata & SEF, CL & on wet soil in ditches & rare \\
\hline 8 & M. rubra & SEF, CL & under decayed leaves & rare \\
\hline 9 & Ramanella montana & SEF, CL & on wet soil near stagnant water pools & rare \\
\hline 10 & Euphlyctis cyanophlyctis & MDF, CL & floats on water surface & very common \\
\hline 11 & E. hexadactylus & $\mathrm{CL}$ & floats and hides among submerged plants in ponds and paddy fields & common \\
\hline 12 & Hoplobatrachus crassus & $\mathrm{CL}$ & on wet soil & rare \\
\hline 13 & H. tigerinus & $C L$ & on banks of canals, ponds and paddy fields & common \\
\hline 14 & Indirana beddomii & MDF, SEF & forest floor-on leaf litter & very common \\
\hline 15 & Fejervarya keralensis & MDF, SEF & stream banks -on wet soil and leaf litter & very common \\
\hline 16 & F. limnocharis & CL, MDF & among grasses and under leaf litter & common \\
\hline
\end{tabular}




\begin{tabular}{llll}
\hline SI. No. Species & Habitat & Microhabitat \\
\hline 17 & Micrixalus fuscus & SEF & on boulders in streams \\
18 & Nyctibatrachus major & SEF & under stones in streams \\
19 & Rana curtipes & SEF & on wet soil and among grasses \\
20 & R. malabarica & $\mathrm{CL}$ & under stones and in crevices \\
21 & R. temporalis & $\mathrm{SEF}, \mathrm{MDF}, \mathrm{CL}$ & on stream banks and in marshes \\
22 & Philautus leucorhinus & $\mathrm{CL}$ & on herbs \\
23 & P. signatus & $\mathrm{CL}$ & on herbs \\
24 & Polypedates maculatus & $\mathrm{CL}$ & on shrubs and trees \\
25 & P. pseudocruciger & $\mathrm{CL}$ & on shrubs and trees \\
26 & Rhacophorus malabaricus & $\mathrm{MDF}$ & on shrubs and trees
\end{tabular}

d) Alappuzha district $\left(90^{\circ} 5^{\prime}-90^{\circ} 52^{\prime} \mathrm{N} \& 7^{\circ} 17^{\prime}-76^{\circ} 48^{\prime} \mathrm{E}\right)$

$\begin{array}{ll}1 & \text { Bufo melanostictus } \\ 2 & \text { B. microtympanum } \\ 3 & \text { B. parietalis } \\ 4 & \text { Microhyla ornata } \\ 5 & \text { M. rubra } \\ 6 & \text { Ramanella variegata } \\ 7 & \text { Euphlyctis cyanophlyctis } \\ 8 & \text { E. hexadactylus } \\ 9 & \text { Hoplobatrachus tigerinus } \\ 10 & \text { Fejervarya limnocharis } \\ 11 & \text { Rana aurantiaca } \\ 12 & \text { R. malabarica } \\ 13 . & \text { Philautus leucorhinus } \\ 14 & \text { Polypedates maculatus } \\ 15 & \text { Rhacophorus malabaricus }\end{array}$

$\begin{array}{ll}\mathrm{CL}, \mathrm{PF} & \text { on bare soil, in crevices and under } \\ \mathrm{CL} & \text { under stones } \\ \mathrm{CL} & \text { on bare soil and under stones } \\ \mathrm{CL} & \text { in water of small ditches } \\ \mathrm{CL} & \text { under decayed litter } \\ \mathrm{CL} & \text { on bare soil } \\ \mathrm{CL} & \text { in water } \\ \mathrm{CL}, \mathrm{PF} & \text { in water- among submerged plants } \\ \mathrm{CL} & \text { in water or wet areas close to water } \\ \mathrm{PF} & \text { among grass } \\ \mathrm{CL} & \text { in water } \\ \mathrm{CL} & \text { under stones or in damp places } \\ \mathrm{CL} & \text { on herbs } \\ \mathrm{CL} & \text { on trees } \\ \mathrm{CL} & \text { on shrubs }\end{array}$

common
rare
rare
rare
rare
rare
common
common
common
very common
rare
rare
common
rare
rare

e) Kottayam district $\left(90^{\circ} 15^{\prime}-10^{\circ} 21^{\prime} \mathrm{N} \& 76^{\circ} 22^{\prime}-77^{\circ} 25^{\prime} \mathrm{E}\right)$

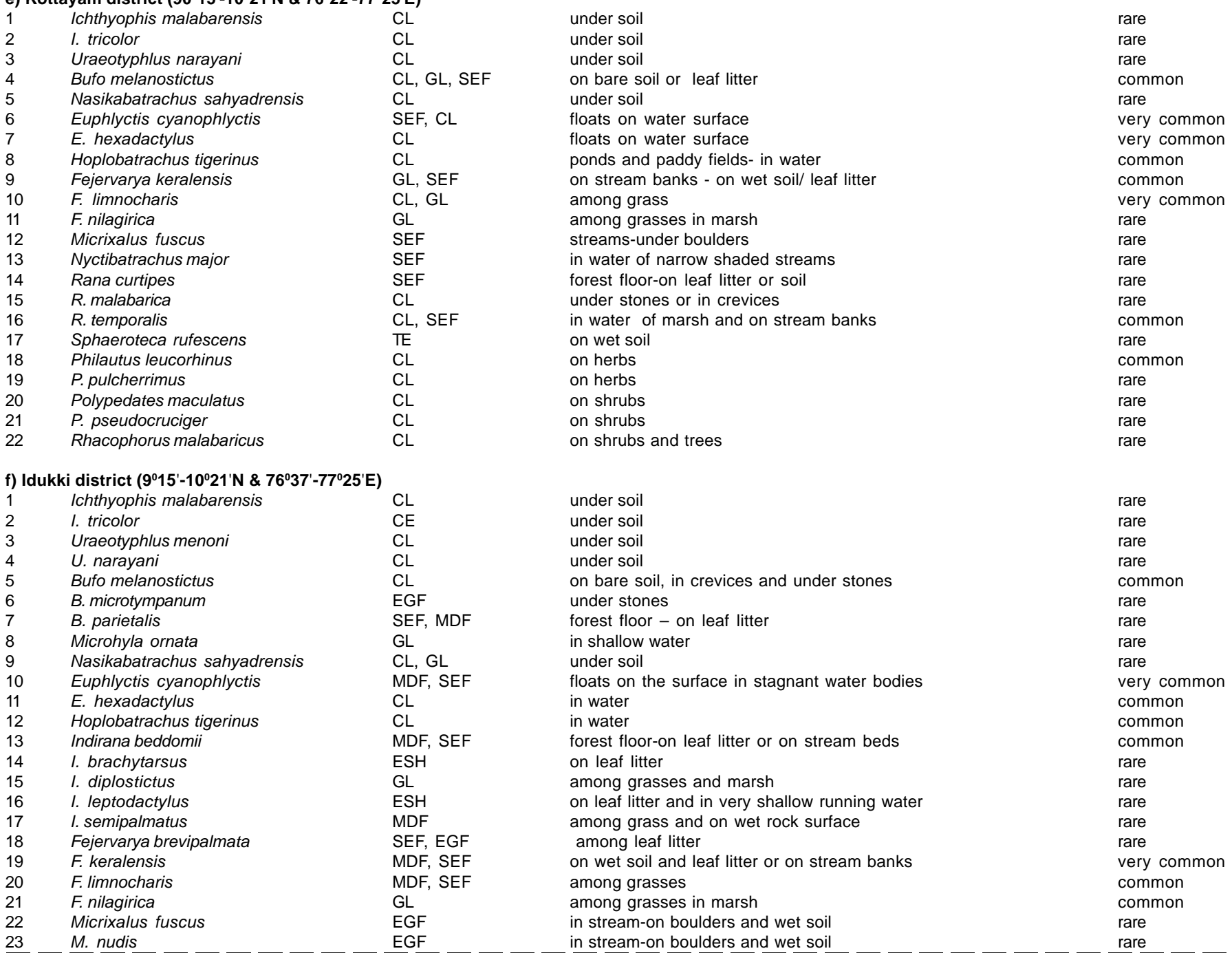




\begin{tabular}{|c|c|c|c|c|}
\hline \multicolumn{2}{|c|}{ SI. No. Species } & \multirow{2}{*}{$\begin{array}{l}\text { Habitat } \\
\text { EGF, SEF }\end{array}$} & \multirow{2}{*}{$\begin{array}{l}\text { Microhabitat } \\
\text { in water }\end{array}$} & \multirow{2}{*}{$\begin{array}{l}\text { Status } \\
\text { common }\end{array}$} \\
\hline 24 & Nyctibatrachus major & & & \\
\hline 25 & N. minor & DEF & under decayed leaf litter & rare \\
\hline 26 & Rana aurantiaca & SEF & in water or on wet soil & rare \\
\hline 27 & R. curtipes & MDF, SEF & forest floor - on leaf litter or bare soil & common \\
\hline 28 & R. malabarica & SEF & on wet soil & rare \\
\hline 29 & Rana temporalis & DDF, CL, ESH & stream sides (on wet soil) and on rocks in streams & common \\
\hline 30 & Sphaeroteca rufescens & MDF & on wet soil in streams & rare \\
\hline 31 & Philautus beddomii & EGF & on herbs & rare \\
\hline 32 & P. femoralis & TE & on herbs & rare \\
\hline 33 & P. leucorhinus & $\mathrm{CL}, \mathrm{MDF}$ & on leaves of herbs and shrubs & common \\
\hline 34 & P. nasutus & SEF & on herbs & rare \\
\hline 35 & P. pulcherrimus & TE & on herbs & rare \\
\hline 36 & $P$. signatus & $\mathrm{GL}$ & on herbs & rare \\
\hline 37 & P. wynaadensis & MDF & on herbs & rare \\
\hline 38 & Polypedates maculatus & MDF & on shrubs or trees & rare \\
\hline 39 & P. pseudocruciger & CL, MDF & on trees & rare \\
\hline 40 & Rhacophorus malabaricus & $\mathrm{CL}$ & on leaves and stems of shrubs and trees & common \\
\hline 41 & Micrixalus gadgili & - & - & - \\
\hline 42 & Rhacophorus lateralis & - & - & - \\
\hline \multicolumn{5}{|c|}{ g) Ernakulam district $\left(90^{\circ} 42^{\prime}-10^{\circ} 18^{\prime} \mathrm{N} \& 76^{\circ} 12^{\prime}-6^{\circ} 42^{\prime} \mathrm{E}\right)$} \\
\hline 1 & Ichthyophis peninsularis & $\mathrm{CL}^{\prime}$ & under soil & rare \\
\hline 2 & l. tricolor & $\mathrm{CL}$ & under soil & rare \\
\hline 3 & Uraeotyphlus interruptus & CL & under soil & rare \\
\hline 4 & U. menoni & $\mathrm{CL}$ & under soil & rare \\
\hline 5 & U. narayani & MDF & inside decaying logs & rare \\
\hline 6 & Bufo melanostictus & TP, MDF & on bare soil and leaf litter & very common \\
\hline 7 & B. microtympanum & TP & on bare soil & rare \\
\hline 8 & B. scaber & $\mathrm{OP}$ & on leaf litter and bare soil & rare \\
\hline 9 & Microhyla ornata & SEF & forest floor - under leaf litter & rare \\
\hline 10 & Euphlyctis cyanophlyctis & TP, MDF & in water & common \\
\hline 11 & E. hexadactylus & SEF & in water & common \\
\hline 12 & Hoplobatrachus tigerinus & TP, MDF & in water & rare \\
\hline 13 & Indirana beddomii & OP, MDF, SEF & forest floor-on leaf litter and under stones & very common \\
\hline 14 & I. brachytarsus & SEF & forest floor-on leaf litter & rare \\
\hline 15 & Fejervarya keralensis & TP, SEF & stream banks-on wet soil or under leaf litter & very common \\
\hline 16 & F. limnocharis & MDF & forest floor - on leaf litter and among grasses & common \\
\hline 17 & Micrixalus nudis & SEF & under stones in dried up streams & rare \\
\hline 18 & Nyctibatrachus major & SEF & in streams-submerged in water or on wet soil surface & rare \\
\hline 19 & N. minor & MDF & under stones in very narrow streams & rare \\
\hline 20 & Rana aurantiaca & MDF & stream banks - on wet soil & rare \\
\hline 21 & R. malabarica & $\mathrm{CL}$ & under soil & rare \\
\hline 22 & R. temporalis & TP, MDF, SEF & in streams-on wet soil and boulders; on roots of river bank trees & very common \\
\hline 23 & Sphaeroteca rufescens & MDF & in wet soil & rare \\
\hline 24 & Philautus beddomii & MDF & on herbs & rare \\
\hline 25 & P. leucorhinus & TP & on leaves of herbs & common \\
\hline 26 & P. temporalis & MDF & on herbs and shrubs & rare \\
\hline 27 & P. wynaadensis & SEF & on herbs & rare \\
\hline \multicolumn{5}{|c|}{ h) Thrissur district $\left(10^{0} 222^{\prime}-10^{0} 26^{\prime} N \& 76^{0} 18^{\prime}-76^{0} 28^{\prime} E\right)$} \\
\hline 1 & Bufo melanostictus & MDF & on bare soil & common \\
\hline 2 & B. scaber & MDF & among grasses in marsh & rare \\
\hline 3 & Microhyla rubra & SEF & streams-in water & rare \\
\hline 4 & Ramanella montana & MDF & forest floor - on wet soil & rare \\
\hline 5 & Euphlyctis cyanophlyctis & MDF, CL & floats in water & very common \\
\hline 6 & E.hexadactylus & MDF & among weeds of shallow ponds & common \\
\hline 7 & Hoplobatrachus crassus & MDF & among grasses in marsh & rare \\
\hline 8 & H. tigerinus & MDF & in or near water bodies & rare \\
\hline 9 & Indirana beddomii & MDF, SEF & forest floor -on leaf litter & common \\
\hline 10 & Fejervarya keralensis & $\mathrm{MDF}, \mathrm{CL}$ & on bare soil and leaf litter & common \\
\hline 11 & F. limnocharis & MDF & forest floor - among grasses & common \\
\hline 12 & F. nilagirica & MDF & among grasses in marsh & rare \\
\hline 13 & Micrixalus fuscus & SEF & streams-on wet boulders and logs & rare \\
\hline 14 & M. nudis & MDF & in water & rare \\
\hline 15 & Nyctibatrachus major & SEF & streams-in water & common \\
\hline 16 & Rana temporalis & MDF, SEF & stream banks-on wet soil and under logs & common \\
\hline 17 & Sphaeroteca rufescens & MDF & in wet soil near water bodies & rare \\
\hline 18 & Philautus leucorhinus & MDF & on herbs & common \\
\hline 19 & Polypedates maculatus & MDF & on shrubs & rare \\
\hline \multicolumn{5}{|c|}{ i) Palakkad district $\left(10^{\circ} 46^{\prime}-10^{\circ} 59^{\prime} N \& 76^{\circ} 28^{\prime}-76^{\circ} 39^{\prime} E\right)$} \\
\hline 1 & Uraeotyphlus oxyurus & EGF & under stones & rare \\
\hline 2 & Ansonia rubigina & EGF & on rocks in rivers & rare \\
\hline 3 & Bufo melanostictus & EGF, GL & on bare soil & very common \\
\hline 4 & B. microtympanum & EGF & on leaf litter & rare \\
\hline 5 & B. parietalis & EGF & on leaf litter and bare soil & rare \\
\hline
\end{tabular}




\begin{tabular}{ll}
\hline SI. No. Species \\
\hline 6 & Ramanella montana \\
7 & R. variegata \\
8 & Euphlyctis cyanophlyctis \\
10 & Indirana beddomii \\
11 & I. leptodactylus \\
12 & Fejervarya keralensis \\
13 & F. limnocharis \\
14 & Micrixalus fuscus \\
15 & M. nudis \\
16 & M. saxicola \\
17 & Nyctibatrachus aliciae \\
18 & N. major \\
19 & N. minor \\
20 & Rana curtipes \\
21 & R. temporalis \\
22 & Sphaeroteca rolandae \\
23 & S. rufescens \\
24 & Philautus chalazodes \\
25 & P. leucorhinus \\
26 & P. nasutus \\
27 & P.pulcherrimus \\
28 & P. signatus \\
29 & P. wynaadensis \\
30 & Polypedates maculatus \\
31 & P. pseudocruciger \\
32 & Rhacophorus malabaricus \\
33 & Ichthyophis longicephalus* \\
34 & Bufo silentvalleyensis \\
35 & Pedostibes tuberculosus \\
36 & Nannobatrachus beddomii \\
37 & Micrixalus thampit \\
38 & Ramanella triangularis \\
39 & Sphaeroteca parambikulamana \\
& \\
& \\
& \\
& \\
& \\
&
\end{tabular}

j) Malappuram district $\left(10^{\circ}\right.$ to $20^{\circ} \mathrm{N} \& 75^{\circ}$ to $\left.77^{\circ} \mathrm{E}\right)$

$\begin{array}{ll}1 & \text { Uraeotyphlus narayani } \\ 2 & \text { Bufo melanostictus } \\ 3 & \text { Euphlyctis cyanophlyctis } \\ 4 & \text { E. hexadactylus } \\ 5 & \text { Hoplobatrachus tigerinus } \\ 6 & \text { Fejervarya brevipalmata } \\ 7 & \text { F. keralensis } \\ 8 & \text { F. limnocharis } \\ 9 & \text { F. syhadrensis } \\ 10 & \text { Ranatemporalis } \\ 11 & \text { Philautus leucorhinus } \\ 12 & \text { P. wynaadensis } \\ 13 & \text { Polypedates pseudocruciger }\end{array}$

Habitat
EGF
CL
TE
EGF
MDF
EGF, MDF
EGF
SEF
EGF
EGF
EGF
EGF, SEF
MDF
MDF
EGF, SEF, MDF
CL
SEF
MDF
MDF
GL inside EGF
EGF
GL inside EGF
EGF
CL
CL
EGF
-
-
-
-
-
-
-

Microhabitat
under stones
on bare soil
in water
forest floor-on leaf litter
on leaf litter
stream banks-on wet soil and leaf litter
among grasses
in streams-on boulders
under boulders and wet rocks
on boulders
under water and on wet soil
in streams - in water or under boulders
in streams -under pebbles
on leaf litter
on wet soil, leaf litter and boulders; in mud crevices of stream banks
on bare soil
on wet soil
on herbs
on herbs
on herbs
on shrubs
on herbs
on shrubs
on shrubs
on shrubs
on trees/shrubs
-
-
-
-
-
-
-

\section{Status}

rare

rare

common

rare

rare

rare

rare

are

rare

rare

rare

common

rare

rare

rare

rare

rare

rare

rare

rare

rare

rare

rare

are

$C L$ inside MDF

TP, MDF

CL, MDF

CL

MDF, TP

MDF

MDF

TP

MDF

MDF

TP

$\mathrm{CL}$

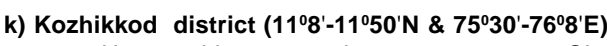

$\begin{array}{lll}1 & \text { Uraeotyphlus narayani } & \mathrm{CL} \\ 2 & \text { Bufo melanostictus } & \mathrm{CL}, \mathrm{SEF} \\ 3 & \text { Euphlyctis cyanophlyctis } & \mathrm{CL} \\ 4 & \text { E. hexadactylus } & \mathrm{CL} \\ 5 & \text { Hoplobatrachus tigerinus } & \mathrm{CL} \\ 6 & \text { Indirana beddomii } & \mathrm{SEF} \\ 7 & \text { Fejervarya keralensis } & \mathrm{SEF} \\ 8 & \text { F. limnocharis } & \mathrm{CL} \\ 9 & \text { Rana temporalis } & \mathrm{CL}, \mathrm{SEF} \\ 10 & \text { Philautus leucorhinus } & \mathrm{CL} \\ 11 & \text { Polypedates pseudocruciger } & \mathrm{CL}\end{array}$

under soil

are

forest floor-on leaf litter and under stones

common

in stagnant water of ponds and pools

in water of ponds/paddy fields

paddy fields-in wet soil

forest floor - among grasses

forest floor-on stream banks

forest floor-among grasses

among grasses near stagnant water pools

streams or marshes-in water or on banks

on leaves of shrubs

on herbs

on shrubs

rare

under soil

on bare soil and leaf litter

in water

in water

in water - among submerged plants

forest floor-on leaf litter and under stones

in streams - on sand or under stones

among grasses or leaf litter

near water bodies-on banks and leaf litter or under boulders

on herbs

on shrubs rare

common

very common

very common

common

rare

common

common

common

rare

rare

I) Wayanad district $\left(11^{0} 27^{\prime}-15^{\circ} 58^{\prime} \mathrm{N} \& 7^{\circ} 47^{\prime}-7^{\circ} 27^{\prime} \mathrm{E}\right)$

Ichthyophis beddomei

Uraeotyphlus menoni

$U$. narayani

Ansonia rubigina

Bufo melanostictus

B. microtympanum

B. parietalis

Kaloula taprobanica

Microhyla ornata

M. rubra

Ramanella montana

$R$. triangularis

Euphlyctis cyanophlyctis
$\mathrm{MDF}, \mathrm{CL}$

$\mathrm{MDF}, \mathrm{CL}$

MDF, CL

ESH, FS

MDF, SEF, CL

MDF, CL

SEF, SH, FS

MDF, CL

$\mathrm{CL}$

CL

FS, COP

MDF, SEF, CL under soil

under soil

under soil

in streams-on or under boulders

on bare soil or leaf litter; in crevices and under stones

on leaf litter

on leaf litte

in puddles

ploughed paddy fields; in the grass/water

ploughed paddy fields; in the grass/water

in termite mounts or on leaf litter

on leaf litter

in water rare

rare

rare

rare

common

rare

common

rare

common

rare

rare

common

very commo 


\begin{tabular}{|c|c|c|c|c|}
\hline \multicolumn{2}{|c|}{ SI. No. Species } & \multirow{2}{*}{$\begin{array}{l}\text { Habitat } \\
\mathrm{CL}, \mathrm{PF}\end{array}$} & \multirow{2}{*}{$\begin{array}{l}\text { Microhabitat } \\
\text { paddy fields and ponds - in water }\end{array}$} & \multirow{2}{*}{$\begin{array}{l}\text { Status } \\
\text { rare }\end{array}$} \\
\hline 14 & E. hexadactylus & & & \\
\hline 15 & Hoplobatrachus tigerinus & $C L, P F$ & on wet soil or mud; in crevices near water & rare \\
\hline 16 & Indirana beddomii & SEF, MDF, TP & on bare soil, leaf litter and under stones & very common \\
\hline 17 & Fejervarya brevipalmata & $\mathrm{MDF}, \mathrm{CL}$ & among grasses or leaf litter & rare \\
\hline 18 & F. keralensis & MDF, TP, SEF, CL & on leaf litter or on wet soil & very common \\
\hline 19 & F. limnocharis & MDF, TP, CL & forest floor-among grasses and leaf litter and in marshy areas & very common \\
\hline 20 & F. nilagirica & MDF & in marsh -among grasses & common \\
\hline 21 & Micrixalus nudis & SEF & on and under rocks/ boulders in streams & rare \\
\hline 22 & Micrixalus phyllophilus & $\mathrm{ESH}$ & on boulders in streams & rare \\
\hline 23 & M. saxicola & ESH, EGF & in streams-on boulders & common \\
\hline 24 & M. silvaticus & ESH & in streams-on boulders & rare \\
\hline 25 & Nyctibatrachus aliciae & $\mathrm{CE}$ & in marsh-under water or litter or on wet soil & rare \\
\hline 26 & N. beddomii & SEF, EGF & in marsh-in water; under litter or stones & rare \\
\hline 27 & N. major & MDF, SEF & in streams-under water or boulders & very common \\
\hline 28 & N. minor & SEF & in marsh-under litter & rare \\
\hline 29 & Rana curtipes & MDF, TP, SEF & forest floor-on bare soil or litter & common \\
\hline 30 & R. temporalis & MDF, SEF, TP, CL & stream banks-on wet soil, litter and in water; among the roots of trees & very common \\
\hline 31 & Philautus chalazodes & $\mathrm{ESH}$ & forest floor - on leaf litter & rare \\
\hline 32 & P. charius & $\mathrm{CL}$ & on herb/shrubs & rare \\
\hline 33 & P. leucorhinus & MDF, CL & on herbs and shrubs & common \\
\hline 34 & P. pulcherrimus & MDF, CL & on herbs & rare \\
\hline 35 & $P$. signatus & $\mathrm{CL}$ & on shrubs & rare \\
\hline 36 & P. tinniens & $\mathrm{SH}$ & on shrubs & rare \\
\hline 37 & P. wynaadensis & MDF, CL & on leaf or trunk of trees & common \\
\hline 38 & Polypedates maculatus & $\mathrm{FS}, \mathrm{CL}$ & on leaf or trunk of trees & rare \\
\hline 39 & P. pseudocruciger & $\mathrm{MDF}, \mathrm{CL}$ & on trees and herbs & rare \\
\hline 40 & Rhacophorus lateralis & $\mathrm{CL}$ & on shrubs & rare \\
\hline 41 & R. malabaricus & MDF, FS & on bamboo stems and leaves & common \\
\hline 42 & R. pleurostictus & - & - & - \\
\hline \multicolumn{5}{|c|}{ 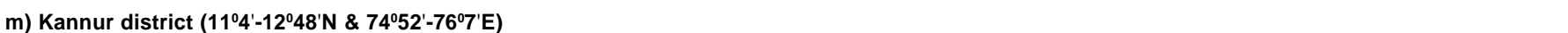 } \\
\hline 1 & Ichthyophis malabarensis & SEF & under soil & rare \\
\hline 2 & I. tricolor & SEF & under soil & rare \\
\hline 3 & Uraeotyphlus menoni & SEF & under soil & rare \\
\hline 4. & Bufo melanostictus & SEF & on leaf litter & rare \\
\hline 5 & Euphlyctis cyanophlyctis & SEF & on the surface of stagnant waters & common \\
\hline 6 & E. hexadactylus & SEF, CL & in water & rare \\
\hline 7 & Hoplobatrachus tigerinus & MDF, SEF, CL & in marshy areas & rare \\
\hline 8 & Indirana beddomii & SEF & among leaf litter in forest floor & rare \\
\hline 9 & Fejervarya brevipalmata & SEF & among grasses in forest floor & rare \\
\hline 10 & F. keralensis & SEF & in streams - under boulders, and in soil or water & common \\
\hline 11 & F. limnocharis & MDF, SEF & on leaf litter and among grasses & rare \\
\hline 12 & Micrixalus nudis & SEF & under or on boulders in streams & rare \\
\hline 13 & M. saxicola & SEF & in streams-on boulders & rare \\
\hline 14 & Nyctibatrachus major & SEF & in streams-under water or rocks & rare \\
\hline 15 & N. minor & SEF & under stones/leaf litter in wet areas & rare \\
\hline 16 & Rana curtipes & SEF & on bare soil & rare \\
\hline 17 & R. temporalis & SEF & in streams - on rocks, logs or wet soil; on stream banks & very common \\
\hline 18 & Sphaeroteca rufescens & MDF & in wet soil & rare \\
\hline 19 & Philautus beddomii & SEF & among leaf litter in forest floor & rare \\
\hline 20 & P. glandulosus & SEF & on leaf of shrubs & rare \\
\hline 21 & P. leucorhinus & SEF & on herbs and shrubs & rare \\
\hline 22 & P. pulcherrimus & SEF & on shrubs/herbs & rare \\
\hline 23 & P. temporalis & SEF & on shrubs and herbs & rare \\
\hline 24 & Polypedates maculatus & SEF & on shrubs and trees & rare \\
\hline 25 & Rhacophorus malabaricus & MDF & on shrubs & rare \\
\hline 26 & Indirana leithii & - & - & - \\
\hline \multicolumn{5}{|c|}{ n) Kasaragod district $\left(12^{0} 5^{\prime}-12^{0} 48^{\prime} N \& 4^{0} 52^{\prime}-7^{\circ} 55^{\prime} E\right)$} \\
\hline 1 & Ichthyophis beddomei & $\mathrm{EGF}, \mathrm{CL}$ & under soil & rare \\
\hline 2 & Uraeotyphlus menoni & MDF & among pebbles and loose gravel & rare \\
\hline 3 & U. narayani & MDF & amidst loose gravel within creeks submerged by water & rare \\
\hline 4 & Bufo melanostictus & EGF, CP & on bare soil or leaf litter & common \\
\hline 5 & B. parietalis & EGF & on humid soil & common \\
\hline 6 & Euphlyctis cyanophlyctis & $\mathrm{FS}, \mathrm{CL}$ & in water & common \\
\hline 7 & Hoplobatrachus crassus & $\mathrm{FS}, \mathrm{CL}$ & in water & rare \\
\hline 8 & H. tigerinus & $\mathrm{CL}$ & among submerged plants in stagnant water bodies & common \\
\hline 9 & Indirana beddomii & EGF, CP & on leaf litter & very common \\
\hline 10 & I. diplostictus & $\mathrm{GL}$ & among grasses in marsh & rare \\
\hline 11 & I. semipalmatus & EGF, MDF & in water & rare \\
\hline 12 & Fejervarya brevipalmata & $\mathrm{CP}$ & among grasses close to streams & rare \\
\hline 13 & F. limnocharis & $\mathrm{CL}$ & among grasses & common \\
\hline 14 & F. keralensis & SEF, TP & stream banks - on wet soil and leaf litter & common \\
\hline 15 & F. syhadrensis & $\mathrm{CP}$ & among grasses and under stones in streams & rare \\
\hline 16 & Micrixalus nudis & MDF & in water & rare \\
\hline
\end{tabular}




\begin{tabular}{|c|c|c|c|c|}
\hline \multicolumn{2}{|c|}{ SI. No. Species } & \multirow{2}{*}{$\begin{array}{l}\text { Habitat } \\
\text { SEF }\end{array}$} & \multirow{2}{*}{$\begin{array}{l}\text { Microhabitat } \\
\text { on wet boulders in streams }\end{array}$} & \multirow{2}{*}{$\begin{array}{l}\text { Status } \\
\text { rare }\end{array}$} \\
\hline 17 & M. saxicola & & & \\
\hline 18 & Nyctibatrachus major & $\mathrm{FS}, \mathrm{CL}$ & under shallow water and rock crevices in streams & common \\
\hline 19 & N. minor & EGF & under boulders in streams & rare \\
\hline 20 & Rana temporalis & $\mathrm{FS}, \mathrm{CL}$ & stream banks-on logs, rocks or wet soil & common \\
\hline 21 & Sphaeroteca rufescens & $\mathrm{CP}$ & on wet soil in streams & rare \\
\hline 22 & Philautus beddomii & $\mathrm{CP}$ & on leaf litter & rare \\
\hline 23 & P. leucorhinus & MDF, EGF, SEF & on herbs and shrubs & very common \\
\hline 24 & P. pulcherrimus & MDF & on shrubs & common \\
\hline 25 & Rhacophorus malabaricus & $\mathrm{CL}$ & on leaf of climbers & rare \\
\hline 26 & Bufo beddomii & - & - & - \\
\hline
\end{tabular}

CE - Cardamom estate; CL - Cultivated land; COP - Coffee plantation; DDF - Dry deciduous forest; DEF - Dry evergreen forest; EGF - Evergreen forest; ESH - Evergreen shola; FS - Forest; GL - Grassland; MDF - Moist deciduous forest; OP - Other plantation; PF - Paddy field; SEF - Semi-evergreen forest; SH - Shola; TE - Tea estate; TP - Teak plantation.

${ }^{*}$ Reported by other workers.

Dubois, A. (1984). La nomenclature supragenerique des Amphibiens Anoures. Memoires du Museum National d'histoire Naturelle (A), 131: 1-64.

Dubois, A., A. Ohler and S.D. Biju (2001). A new genus and species of Ranidae (Amphibia: Anura) from south-west India. Alytes 19(2-4): 53-79

Dubois, A. and A. Ohler (1999). Asian and Oriental toads of the Bufo melanostictus, Bufo scaber and Bufo stejnegeri groups (Amphibia: Anura): a list of available and valid names and redescription of some namebearing types. Journal of South Asian Natural History 4(2): 133-180.

Easa, P.S. (1998). Survey of reptiles and amphibians in Kerala part of Nilgiri Biosphere Reserve. KFRI Research Report No.148, Kerala Forest Research Institute, Peechi, Trichur.

Ferguson, H.S. (1904). A list of Travancore batrachians. Journal of the Bombay Natural History Society 14: 499-509.

George, S. (1995). Amphibian survey at the proposed Pooyamkutty hydro-electric power project site, Kerala. Report of the FRAWG Project: Survey of Amphibians of Kerala 1. Zoos' Print 10(7): 1-4.

George, S., J.M. Samuel and V.S Josekumar (1996). Survey of the amphibian fauna of Thattekkad Bird Sanctuary, Kerala. Zoos' Print 11(5): 7-8.

Inger, R.F., H.B. Shaffer, M. Koshy and R. Bakde (1984a). A report on a collection of amphibians and reptiles from the Ponmudi, Kerala, South India. Journal of the Bombay Natural History Society 81: 406427.

Inger, R.F., H.B. Shaffer, M. Koshy and R. Bakde (1984b). A report on a collection of amphibians and reptiles from the Ponmudi, Kerala, South India. Journal of the Bombay Natural History Society 81(3): 802832.

Iype,V.C., E.V. Nybe and P.V. Pillai (1991). Status report of High Zone. National Agricultural Project, Kerala.

Oommen, V.O., G.J. Measey, W.J. Gower and M. Wilkinson (2000). Distribution and abundance of the caecilian, Gegeneophis ramaswamii (Amphibia: Gymnophiona) in southern Kerala. Current Science 79(9): 1386-1389.

Pillai, R.S. (1978). A new frog of the genus Micrixalus Boul. from Wayanad, S. India. Proceedings of the Indian Acadamy of Sciences (B) 87: $173-177$.

Pillai, R.S. (1981). Two new species of amphibia from Silent Valley, S. India. Bulletin of the Zoological Survey of India 3(3):153-158.

Pillai, R.S. (1986). Amphibian fauna of Silent Valley, Kerala. Records of the Zoological Survey of India 84 (1-4): 229-242.

Pillai, R.S. and R. Pattabiraman (1981). A new species of torrent toad (genus: Ansonia) from Silent Valley, S. India. Proceedings of the Indian Acadamy of Sciences (B) 90: 203-208.

Pillai, R.S. and R. Pattabiraman (1990). Amphibians from Sabarigiri forest, Western Ghats, Kerala, including a new species of Micrixalus. Records of the Zoological Survey of India 86(2): 383-390.

Radhakrishnan, C. (1996a). Amphibians from Aralam Wildlife Sanctuary, Western Ghats, Kerala. Zoos' Print 11(8): 1-6.
Radhakrishnan, C. (1996b). Amphibians of the Parambikulam Wildlife Sanctuary, Kerala. Cobra 26: 10-17.

Radhakrishnan, C., P.M. Sureshan and K. Gopi (1996). Taxonomic studies on a collection of fauna from Eravikulam National Park. Paper presented in the symposium on biodiversity conservation of High Ranges. High Range Wildlife and Environment Preservation Association, Munnar, July 1996.

Radhakrishnan, C. and M.S. Ravichandran (1999). First report of the Amphibians of Kasaragod district, Kerala. Records of the Zoological Survey of India 97(3): 17-30.

Ravichandran, M.S. and R.S. Pillai (1990). On the collection of frogs and toads from Periyar Wildlife Sanctuary. Records of the Zoological Survey of India 87(2): 121-126.

Zacharias, V. and A.K. Bhardwaj (1996). A preliminary list of amphibian fauna of Periyar Tiger Reserve, Thekkady, Kerala, South India. Indian Forester 122: 247-249.

\section{ACKNOWLEDGEMENTS}

Our thanks are due to the Ministry of Environment and Forests, Govt. of India, for providing financial assistance to carry out this work as a major research project (No.31/11/98-NE) awarded to M.I.A and S.G. J.J. thanks the Ministry for the fellowship. We are thankful to Kerala Forest Department for access to protected areas and for permissions to collect specimens. We also thank the Principal, Mar Thoma College, Tiruvalla, Kerala for the institutional support during the tenure of the work. The help rendered by S.K. Dutta (Utkal University) and M.S. Ravichandran (ZSI) for the identification of specimens is gratefully acknowledged.

w See images of some Amphibians in the websupplement at www.zoosprint.org

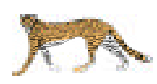

